\title{
Comportamiento de los aerosoles atmosféricos en el Observatorio de Huancayo durante los eventos de quema de biomasa del año 2019
}

\author{
Desar Victoria ${ }^{* 1,2,3}$ y René Estevan ${ }^{4}$ \\ ${ }^{1}$ Universidad Nacional del Callao, Facultad de Ingeniería Ambiental y Recursos Naturales, Callao, Perú \\ ${ }^{2}$ Universidad Nacional del Callao, Facultad de Ciencias Naturales y Matemática, Callao, Perú \\ ${ }^{3}$ Universidad Nacional Mayor de San Marcos, Unidad de Posgrado de Física, Lima, Perú \\ ${ }^{4}$ Instituto Geofísico del Perú, Lima, Perú \\ Recibido 05 enero 2021 - Aceptado 24 abril 2021
}

\begin{abstract}
Resumen
Se determinaron las características del aerosol recuperado en la estación AERONET de Huancayo. El estudio se realizó durante los eventos de quema de biomasa del año 2019. Se procesaron los datos del sensor MODIS de los satélites Aqua y Terra, y del fotómetro CIMEL. Se han calculado los valores medios, mínimos y máximos del espesor óptico del aerosol (AOD) y el exponente de Angstrom (EA), asimismo se determinaron los tipos de aerosoles presentes en el Observatorio de Huancayo. Para la intercomparación de los resultados se utilizaron los datos del Fotómetro de la red internacional AERONET localizado en Huancayo. La media del AOD y el EA medido con AERONET respectivamente es $0.159 \pm 0.073$ (adimensional) y $1.624 \pm 0.198$ (adimensional). Los valores medios del AOD Aqua y Terra son respectivamente $0.088 \pm 0.056$ y $0.098 \pm 0.087$. El análisis de retrotrayecoria realizado mediante HYSPLIT muestra que la mayor parte de aerosoles que llegan al observatorio de Huancayo provienen de las regiones de Ucayali, Huánuco y Pasco, sin embargo, hay un pequeño porcentaje que se genera en los territorios de Brasil y Bolivia. Al intercomparar el AOD Aqua y Terra con AERONET, se obtuvo respectivamente los coefientes de correlación (R) 0.794 y 0.796.
\end{abstract}

Palabras clave: MODIS, AERONET, AOD y EA.

\section{Behavior of atmospheric aerosols at the Huancayo Observatory during the 2019 biomass burning events}

\begin{abstract}
The characteristics of the aerosol recovered at the Huancayo AERONET station were determined. The study was carried out during the 2019 biomass burning events. Data from the MODIS sensor from the Aqua and Terra satellites and from the CIMEL photometer were processed. The mean, minimum and maximum values of the aerosol optical thickness (AOD) and the Angstrom exponent (EA) have been calculated, as well as the types of aerosols present in the Huancayo Observatory. For the intercomparison of the results, the data from the photometer of the international AERONET network located in Huancayo were used. The mean AOD and EA measured with AERONET respectively is $0.159 \pm 0.073$ (no unit) and $1.624 \pm 0.198$ (no unit). The mean values of the Aqua and Terra AOD are respectively $0.088 \pm 0.056$ and $0.098 \pm 0.087$. The back-trace analysis carried out using HYSPLIT shows that most of the aerosols that arrive at the Huancayo observatory come from the regions of Ucayali, Huánuco and Pasco, however, there is a small percentage that is generated in the territories of Brazil and Bolivia. By intercomparing the AOD Aqua and Terra with AERONET, the correlation coefficients (R) 0.794 and 0.796 were obtained respectively.
\end{abstract}

Keywords: MODIS, AERONET, AOD and EA.

*cesar.victoria@unmsm.edu.pe 


\section{Introducción}

Los aerosoles son partículas sólidas o líquidas suspendidas en la atmósfera cuyos tamaños pueden variar entre $0.001 \mu \mathrm{m}-100 \mu \mathrm{m}$ (Mao et al., 2018) [Mao18], tienen origen natural o antropogénico (Boiyo et al., 2017) [Boi17]. Los aerosoles afectan directamente el equilibrio de radiación de la Tierra y el clima dispersando y absorbiendo la radiación solar, e indirectamente cambiando las propiedades microfísicas de las nubes (Xu et al, 2020) [Xu20], lo cual conduce a lo modificación de las eficiencias de la precipitación y de los perfiles de temperatura y humedad (Stefan et al., 2020) [Ste20]. Los aerosoles contaminan el aire, crean problemas de salud (Xu et la., 2020) y disminuyen la visibilidad y puede dar lugar a condiciones desfavorables para el transporte (Boiyo et al., 2017) [Boi17].

En la Amazonía existe una gran presión económica para convertir bosques en tierras agrícolas y ganaderas (Scanes, 2018) [Sca18], para este fin se han deforestado miles de hectáreas de bosques. Como consecuencia de la deforestación de la Amazonía se quema la biomasa, por lo que se produce emisión de aerosoles, las comunidades rurales de las regiones de Junín, Pasco, Huánuco y Ucayali, dependen de la quema de Biomasa para sus necesidades energéticas diarias (Bhatt et al, 2017) [Bha17]. La mayor quema de biomasa se produce entre mediados de julio y octubre (Estevan et al, 2019) [Est19]. Por otra parte, la minería ilegal convierte los bosques en desiertos (Osores et al., 2012) [Oso12] ocasionando la emisión del polvo a la atmósfera. Los aerosoles generados en la Amazonía son transportados por los vientos alisios hacia el valle del Mantaro ( Bhat et al, 2017) [Bha17]. El $97.7 \%$ de los aerosoles que provienen de la quema de biomasa (BIO) se originan en el Perú y el $2.3 \%$ se origina en Brasil y Bolivia (Moya et al, 2017) [Moy17].

Los aerosoles tienen características regionales propias, ya que tienen una gran variación tanto en el tiempo, como en el espacio; lo cual hace que haya incertidumbre respecto al rol que cumplen en el cambio climático (IPCC, 2013) [IPC13]. Esta incertidumbre insta a investigar las propiedades físicas y químicas a nivel local, regional y global (Xu, et al, 2020) [Xu20]. Los estudios sobre las propiedades de los aerosoles, su variabilidad espacial y temporal en relación con los procesos que experimentan en la atmósfera ayudan a mejorar el conocimiento sobre el clima cambiante que nos enfrentamos actualmente (Stefan et al., 2020) [Ste20].

Los aerosoles se caracterizan mediante los siguientes parámetros: profundidad óptica del aerosol (AOD) y el exponente de Angstrom (EA). El AOD es una medida de la distribución del aerosol dentro de una columna de aire desde la superficie de la tierra hasta la parte superior de la atmósfera y constituye el principal parámetro utilizado para evaluar la carga del aerosol en la atmósfera (Boiyo et al, 2017) [Boi17]. EA se utiliza para describir la de- pendencia espectral de AOD en una longitud de onda, el EA es un parámetro que permite determinar el tamaño de las partículas del aerosol.

En la actualidad existen sensores tales como el Radiómetro Avanzado de Muy Alta Resolución (AVHRR), el Espectrómetro de Mapeo de Ozono Total (TOMS) y el Espectroradiómetro de Imágenes de Mediana Resolución (MODIS). Estos sensores proporcionan características espaciales y temporales de los aerosoles tanto a nivel local, regional y global (Flousti et al, 2016; Boiyo et al, 2017) [Flo16] [Boi17]. El sensor MODIS a bordo de los satélites Aqua y Terra tiene una órbita polar, posee 36 bandas espectrales con longitudes de onda que varían desde 0.415 a $14.235 \mu \mathrm{m}$, en cuanto a su resolución espacial posee dos bandas a $205 \mathrm{~m}, 5$ bandas a $500 \mathrm{~m}$ y 29 bandas $1 \mathrm{~km}$. Para el estudio de aerosoles se utilizan las siete primeras bandas que varían entre 0.145 y $2.155 \mu \mathrm{m}$ (Boiyo et al, 2017) [Boi17].

La forma de obtener las propiedades ópticas de los aerosoles, en puntos fijos sobre la superficie terrestre, es mediante los fotómetros solares CIMEL pertenecientes a Aerosol RObotic NeTwork (AERONET). AERONET proporciona información del AOD y del EA. El AOD recuperado por MODIS ha sido intercomparado con el AOD recuperado por AERONET.

El Modelo Híbrido Lagrangiano de Trayectoria Integrada de Partícula Única (HYSPLIT) se utiliza para examinar la trayectoria de masas de aire, con la finalidad de determinar el origen del aerosol (Boiyo et al, 2017) [Boi17].

Se han realizado varios estudios sobre el comportamiento de los aerosoles en Huancayo, tales como Vivanco (2014) [Viv14] y Suarez et al. (2017) [Sua17], pero ningunos de ellos caracterizaron los aerosoles en periodo específico durante la temporada de quema de biomasa. En el presente estudio se han caracterizado los aerosoles en la temporada de quema de biomasa durante el año 2019. El objetivo de este estudio es analizar la variación temporal y espacial del AOD y del EA, determinar los tipos de aerosoles e intercomparar el AOD MODIS con el AOD AERONET en la estación AERONET de Huancayo durante la temporada de la quema de biomasa del año 2019, el periodo de quema de biomasa está comprendido entre los meses de julio y octubre. El artículo está organizado del siguiente modo: en sección 2 se describe la zona de estudio, los datos y la metodología usado en la investigación. En la sección 3 se presentan y se discuten los resultados. Finalmente, en la sección 4 se muestra las conclusiones.

\subsection{Lugar de estudio}

El estudio se realiza en el Laboratorio de Microfísica Atmosférica y Radiación (LAMAR), el cual forma parte del Observatorio de Huancayo, perteneciente al Instituto 
Geofísico del Perú (IGP), que se encuentra ubicado en un área rural a $12 \mathrm{~km}$ al Oeste de la ciudad de Huancayo (12 02 '18" S, $75^{\circ} 19^{\prime} 22^{\prime \prime}$ O, 3350 m.s.n.m).



Figura 1: Ubicación del Observatorio de Huancayo en el Valle del Mantaro.

\section{Datos y metodología}

\subsection{Datos MODIS}

El sensor MODIS se encuentra a bordo de los satélites Aqua y Terra desde diciembre de 1999 y mayo del 2002 respectivamente, ambos satélites pertenecen al Sistema de Observación de la Tierra (Mangla et al., 2020) [Man20]. El satélite Terra cruza el ecuador de Norte a Sur, alrededor de las 10:00 hora local; mientras Aqua se dirige de Sur a Norte alrededor de 13:30 hora local (Wei et al., 2019) [Wei19]. Los productos MODIS son almacenados en diferentes niveles y varias versiones llamados "colecciones" (Boiyo et al., 2017) [Boi17]. La colección 6 (C6) fue elaborado a partir de tres algoritmos que operan en diferentes tipos de superficies. Los dos algoritmos de recuperación de aerosol son: Dark Target (DT) para la recuperación sobre océano (oscuro en el visible y longitudes de onda larga) y sobre tierras con vegetación y/o suelo oscuro (oscuro en el visible), y el tercer algoritmo Deep Blue (DB), desarrollado para la recuperación sobre el desierto y/o tierras raras (brillante en el visible) (Levy et al., 2013) [Lev13]. La colección 6.1 (C6.1) es la versión mejorada de C6, C6.1 agrega un nuevo conjunto de datos que combina DT y DB en un nuevo conjunto de datos llamado DBT (Mangla et al, 2020) [Man20]. En este trabajo de investigación se utilizó la colección 6.1 y nivel 1 , con una resolución espacial de $3 \mathrm{~km}$.

\subsection{Datos AERONET}

AERONET es una red de fotómetros solares para las mediciones de aerosoles. Los sensores miden radiación solar directa en longitudes de onda de 340 a $1020 \mathrm{~nm}$ y radiancias difusas del cielo entre 440 y $1020 \mathrm{~nm}$ (Mangla et al., 2020) [Man20] a una resolución temporal de $15 \mathrm{~min}$ con un sesgo bajo aproximadamente de 0.01 a 0.02 en condiciones sin nubes (Wei et al., 2019) [Wei19]. La red AERONET proporciona base de datos de forma gratuita continua, los cuales se pueden extraer de la siguiente página web (http://aeronet.gsfc.nasa.gov/). AERONET proporciona información de AOD, EA y contenido de agua precipitable (Fernandez et al., 2017) [Fer17]. Los datos AOD se calculan en tres niveles de calidad: Nivel L1.0 (dato original), Nivel L1.5 (filtrado de nubes) y Nivel L2.0 ( postcalibración) (Fernández et al., 2017; Wei et al., 2019) [Fer17] [Wei19]. El producto AERONET que se utilizó en la investigación es la Versión 3 Nivel L2.0.

\subsection{Datos FINN}

El Inventario de Incendios NCAR (FINN), proporciona estimaciones de emisiones globales de alta resolución de gases traza, partículas de quema de biomasa, que incluye incendios forestales e incendios agrícolas, pero no incluye el uso de biocombustible y basura ardiente (Wiedinmyer et al., 2011; Vongruand y Pimonsree, 2020). FINN (http://www2.acom.ucar.edu/modeling/ finn-fire-inventory-ncar) utiliza detecciones diarias de incendios activos MODIS y cobertura terrestre para proporcionar estimaciones de emisiones diarias con una resolución de $1 \mathrm{~km}^{2}$ (Koplitz et al., 2018) [Kop18].

\subsection{Intercomparación del AOD MODIS con AERONET}

Para evaluar la precisión de los valores del AOD MODIS se compararon con las mediciones de AOD de AERONET (Wei et al., 2019) [Wei19]. El AOD AERONET obtenido en una longitud de onda se convierte en AOD MODIS mediante la siguiente ley de potencia (Boiyo et al., 2017) [Boi17]:

$$
A O D_{a}=A O D_{b}\left(\frac{a}{b}\right)^{-E A}
$$

Donde $a=550 \mathrm{~nm}$ es la longitud de onda para la recuperación del AOD MODIS, $b=500 \mathrm{~nm}$ es la longitud de onda para la recuperación del AOD AERONET y EA es el exponente de Angstrom recuperado por AERONET. El criterio espacial y temporal fijado para la intercomparación es $25 \mathrm{~km}$ rango (distancia medida a partir de 
la estación AERONET) y 30 minutos respectivamente (Levy et al., 2013) [Lev13].

\section{Resultados y Discusión}

\subsection{Distribución espacial y temporal del AOD MODIS}

En esta subsección se muestra la distribución espacial y temporal del AOD MODIS medido a $550 \mathrm{~nm}$. Se calcularon los valores medios, mínimos, máximos y desviación estándar $(\sigma)$ del AOD y EA.

En la Figura 2a se muestra la distribución del AOD Aqua sobre el valle del Mantaro, el valor del AOD predominante oscila entre 0.02 y 0.10 , en parte Norte del valle el $A O D$ varía entre 0.06 y 0.08 , en la central varía entre 0.04 y 0.14 y en la parte Sur el AOD oscila entre 0.00 y 0.06; los menores valores del AOD se encuentran en esta zona. En la parte Noreste del valle el AOD alcanza un valor máximo de 0.33 , este resultado puede ser debido a la dirección Noreste del viento predominate sobre el valle del Mantaro, ya que los aerosoles generados en la selva del Perú son transportados por desplazamiento de masas de aire (Estevan et al., 2019) [Est19] y en la parte Este AOD alcanza un valor máximo de 0.23 .

En la Figura 2b se muestra la distribución del AOD Terra sobre el valle del Mantaro, el valor del AOD predominante oscila entre 0.02 y 0.06 , en parte Norte del valle el AOD varía entre 0.00 y 0.12 , en la parte central varía entre 0.00 y 0.16 , y en la parte Sur el AOD oscila entre 0.00 y 0.16 ; los menores valores del AOD se encuentran en la parte central y Sur del valle. En la parte Noreste del valle el AOD alcanza un valor máximo de 0.35 , y en la parte Este AOD alcanza un valor máximo de 0.21.

La Tabla 1 muestra los valores medios, mínimos y máximos del AOD MODIS para mediciones coincidentes un rango de $25 \mathrm{~km}$ tomando como centro el Observatorio de Huancayo y con una coincidencia temporal de $\pm 30 \mathrm{minu}-$ tos, se observa que el que valor medio, mínimo y máximo del AOD Terra es mayor que Aqua; así mismo, el AOD Terra tiene mayor $\sigma$ que Aqua. En la Tabla 2 se muestra los valores mensuales del AOD Aqua, se observa que el mayor valor medio del AOD (0.162) ocurre en el mes de setiembre, sin embargo, el mayor valor máximo absoluto del AOD (0.318) ocurre en el mes de agosto, esto puede ser devido a la mayor cantidad de incendios forestales registrados, tal como se muestra en la Tabla 4. En la Tabla 3 se muestran los valores mensuales del AOD Terra, se observa que el mayor valor medio del AOD (0.204) ocurre en el mes de setiembre, este resultado es debido a que en le mes de setiembre, los árboles talados en el mes de marzo con fines agrícolas ya están muy secos para el mes de setiembre. Sin embargo, el valor máximo abosuluto del AOD (0.391) al igual que en Terra se registró en el mes de agosto.

La Figura 3 muestra la variación temporal del AOD MODIS para las mediciones coincidentes con AERONET. El 19 de agosto el AOD alcanza su mayor valor tanto para Aqua (0.318) como para Terra (0.391), esto puede ser devido a la mayor cantidad de incendios forestales registrados, tal como se muestra en la Tabla 4. Las discontinuidades de los valores AOD MODIS de la Figura 3, es debido a la falta de datos en algunos días del periodo en estudio.

\begin{tabular}{lcccr}
\hline Satélite & \multicolumn{4}{c}{ AOD } \\
& Medias & $\sigma$ & Mínimo & Máximo \\
\hline Aqua & 0.088 & 0.056 & 0.006 & 0.318 \\
Terra & 0.098 & 0.087 & 0.009 & 0.391 \\
\hline
\end{tabular}

Tabla 1: Valores promedio del AOD MODIS en el periodo julio octubre del año 2019.

\begin{tabular}{lcccr}
\hline Mes & Medias & $\sigma$ & Mínimo & Máximo \\
\hline Jul & 0.079 & 0.021 & 0.039 & 0.117 \\
Ago & 0.079 & 0.065 & 0.006 & 0.318 \\
Set & 0.162 & 0.066 & 0.038 & 0.289 \\
Oct & 0.097 & 0.030 & 0.014 & 0.187 \\
\hline
\end{tabular}

Tabla 2: Estadística mensual del AOD Aqua en el periodo julio octubre del año 2019.

\begin{tabular}{lcccr}
\hline Mes & Medias & $\sigma$ & Mínimo & Máximo \\
\hline Jul & 0.057 & 0.029 & 0.009 & 0.178 \\
Ago & 0.200 & 0.099 & 0.012 & 0.391 \\
Set & 0.204 & 0.066 & 0.204 & 0.204 \\
Oct & 0.097 & 0.030 & 0.014 & 0.187 \\
\hline
\end{tabular}

Tabla 3: Estadística mensual del AOD Terra en el periodo julio octubre del año 2019. 


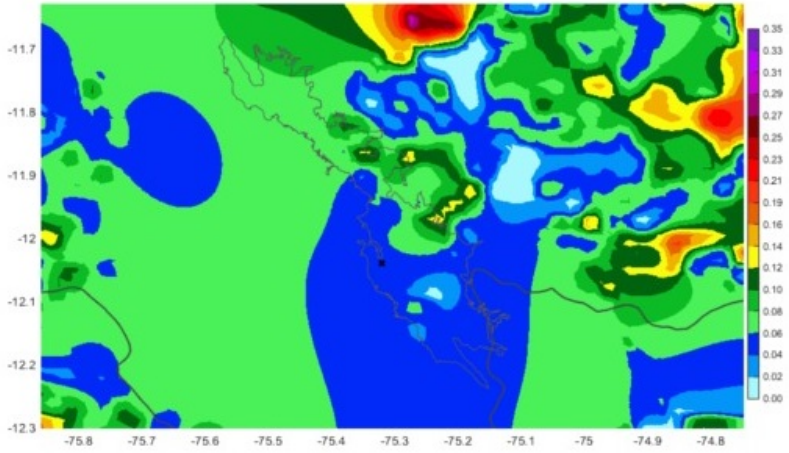

(a) Aqua

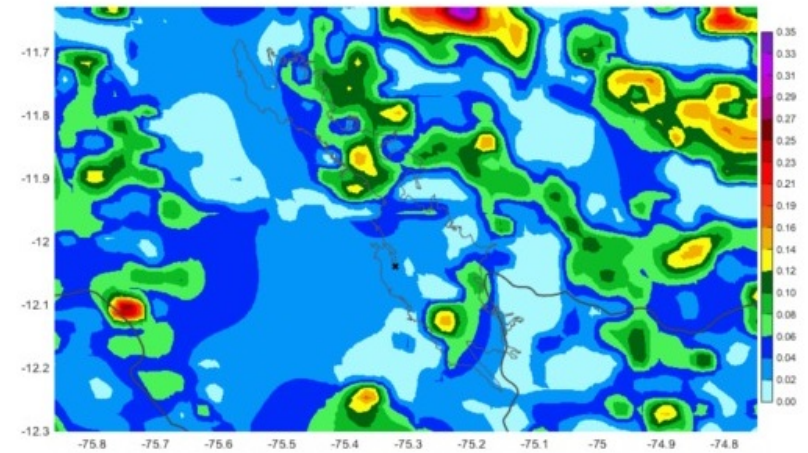

(b) Terra

Figura 2: Distribución del AOD MODIS para el periodo de julio a octubre del 2019.

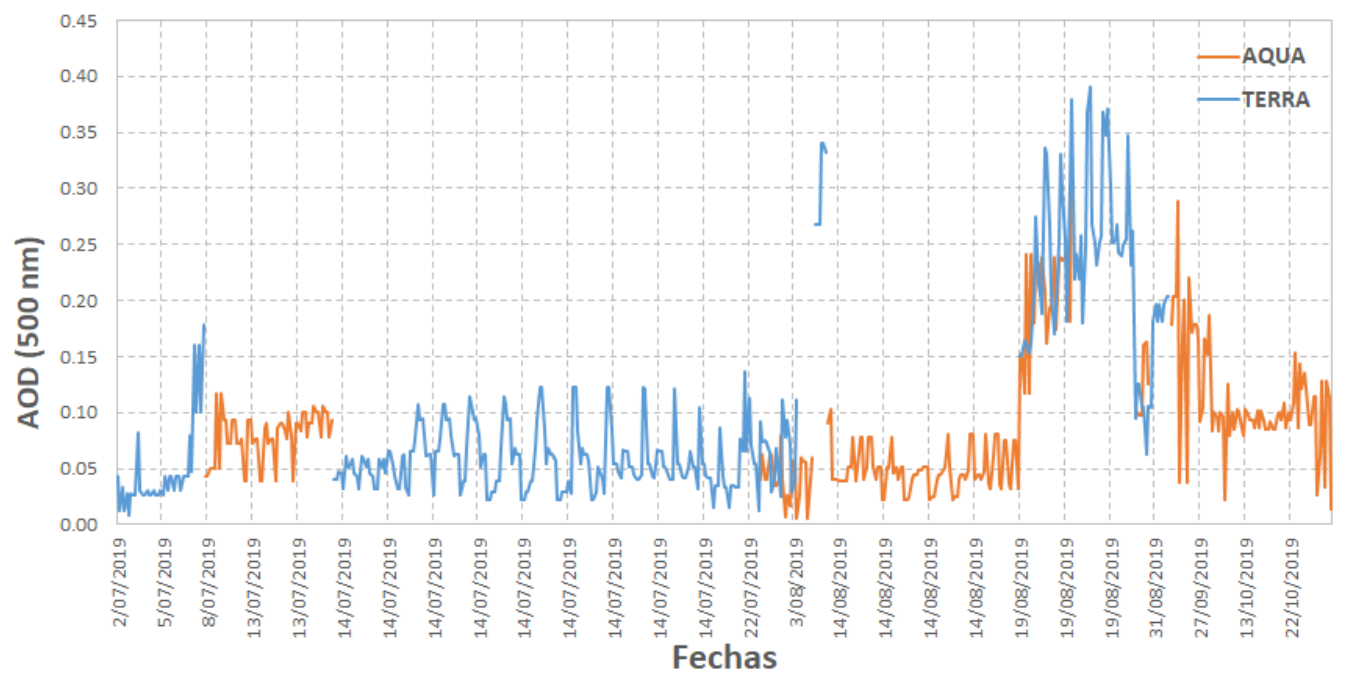

Figura 3: Serie de tiempo de la variación del AOD MODIS obtenido en una longitud de onda $550 \mathrm{~nm}$.

\subsection{Focos de incendios forestales}

En la Tabla 4 y Figura 4 se muestran los focos de incendios forestales con sus respectiva cantidad de emisión de material particulado $P M_{10}$ (partículas con diámetro menor o igual a $10 \mu \mathrm{m}$ ). Se observa que la mayor cantidad de incendios ocurrieron en el mes de agosto (62 629 focos), seguido por setiembre (60952 focos). La menor cantidad de incendios ocurrieron en los meses de octubre (20 941 focos) y julio (11 939 focos). El foco de incendio que generó la mayor cantidad de material particulado $P M_{10}$ se registró en el mes setiembre, con un valor de $9.58 \times 10^{4}(\mathrm{~kg} /$ día $)$, mientras que el foco que generó la menor cantidad de material particulado $P M_{10}$ se registró en el mes de agosto con un valor de $1.10 \times 10^{-1}$ ( $\mathrm{kg} /$ día). Aunque en el mes de agosto se registra el mayor número de focos de incendios forestales, las mayores emisiones se registran en setiembre, esto explica por qué, en el AOD AERONET, los mayores valores del AOD se registran en setiembre, tal como se muestra en la Tabla 6

\begin{tabular}{lcclc}
\hline Mes & NF & \multicolumn{3}{c}{ Emisión de $P M_{10}(\mathrm{~kg} /$ día $)$} \\
& & Medias & \multicolumn{1}{c}{ Mínimo } & Máximo \\
\hline Jul & 11939 & $1.33 \times 10^{4}$ & $1.10 \times 10^{1}$ & $9.55 \times 10^{4}$ \\
Ago & 62629 & $1.83 \times 10^{4}$ & $1.10 \times 10^{-1}$ & $9.57 \times 10^{4}$ \\
Set & 60957 & $1.89 \times 10^{4}$ & $1.01 \times 10^{1}$ & $9.58 \times 10^{4}$ \\
Oct & 20941 & $1.39 \times 10^{4}$ & $1.89 \times 10^{-1}$ & $7.33 \times 10^{4}$ \\
\hline
\end{tabular}

Tabla 4: Incendios forestales registrados en el periodo julio octubre del año 2019. Donde NF es el números de focos de incendios forestales 


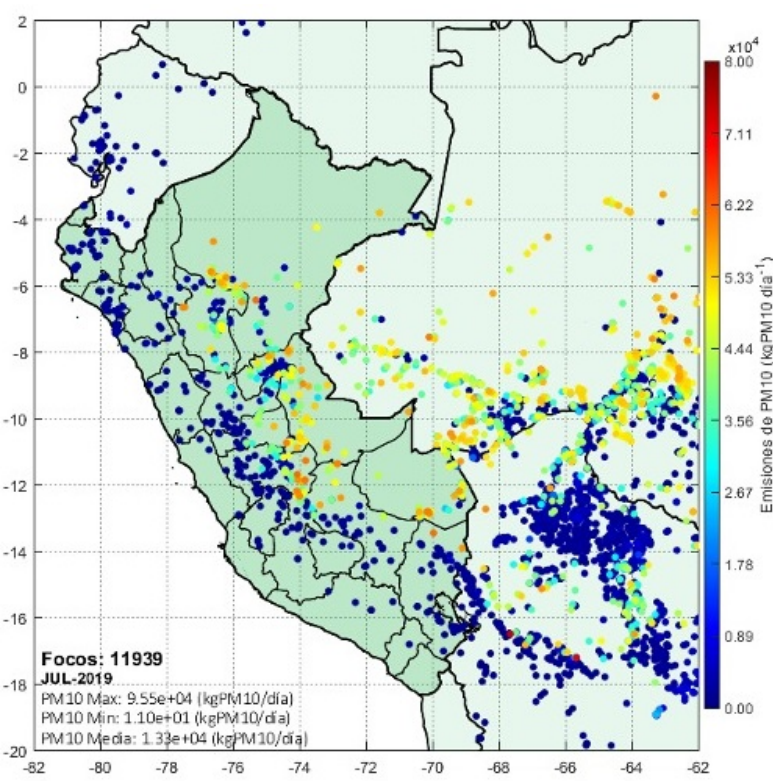

(a) Julio

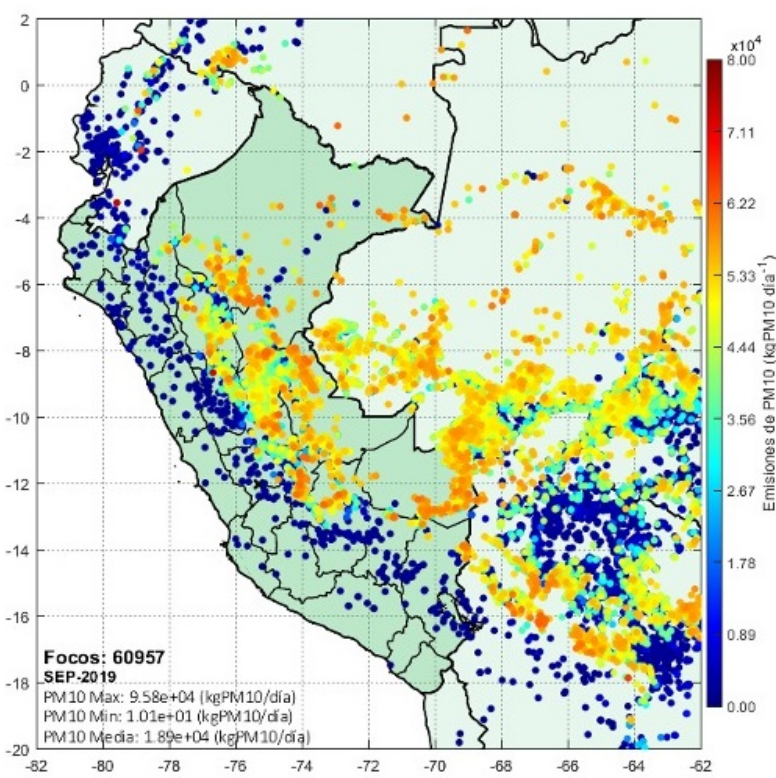

(c) Septiembre

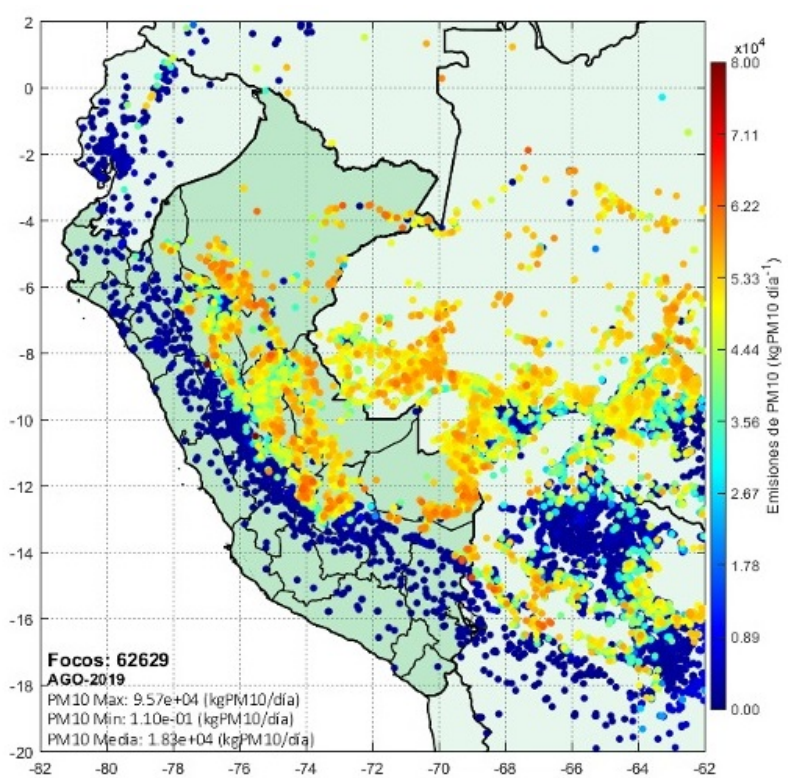

(b) Agosto

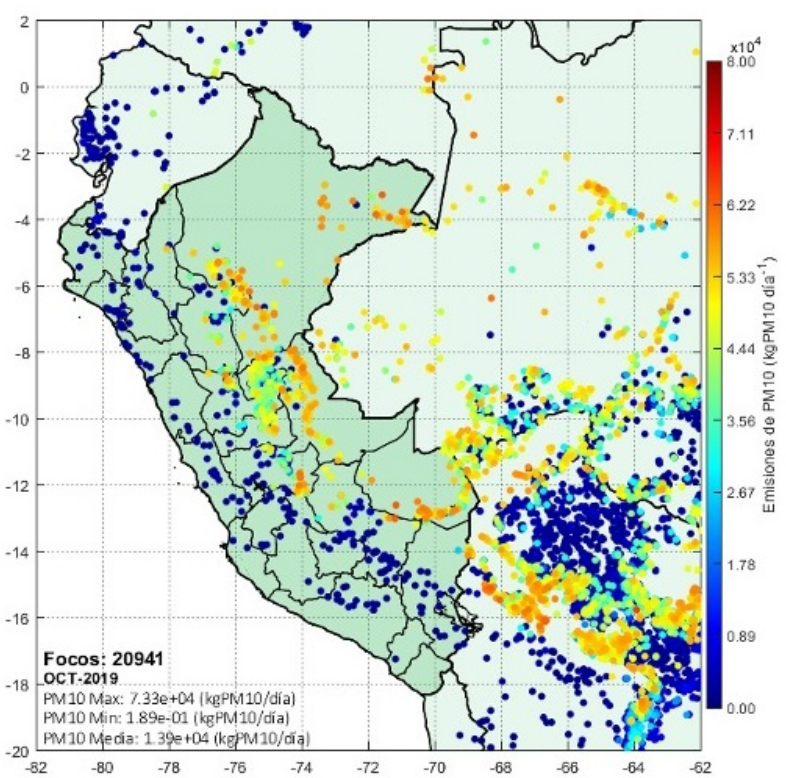

(d) Octubre

Figura 4: Focos de incendios forestales. Los puntos en colores representan los incendios registrados. 


\subsection{Variación temporal del AOD y EA me- didos con AERONET}

En esta subsección se calcularon los valores medios, mínimos, máximos y $\sigma$ del AOD y EA. El AOD fue medi- do a una longitud de onda de $440 \mathrm{~nm}$ y el EA fue medido a una longitud de $440-780 \mathrm{~nm}$.

En la Tabla 5 se observan los valores del AOD y EA obtentidos de AERONET, hay un total de 10029 mediciones para el periodo en estudio.

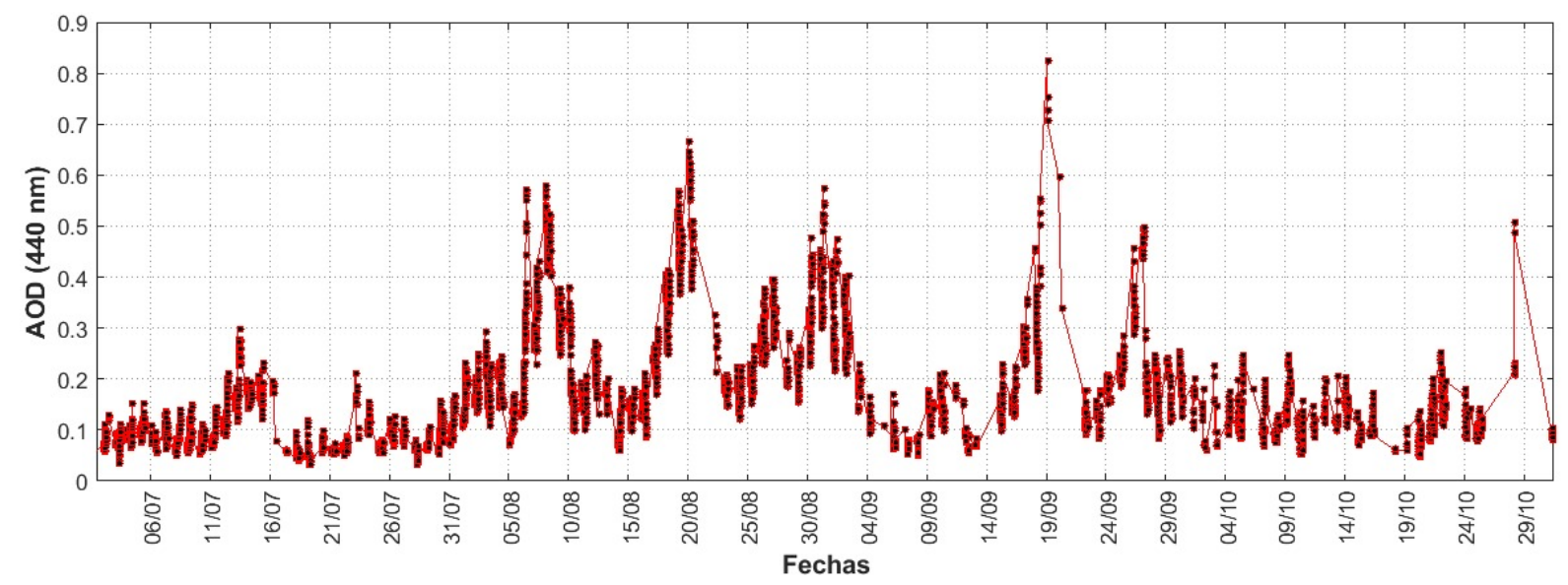

Figura 5: Variación del AOD AERONET.



Figura 6: Variación del EA AERONET.

La Figura 5 muestra la variación temporal del AOD, el mínimo absoluto del AOD (0.031) se registró el 28 de julio y máximo absoluto del AOD (0.825) se registró el 19 de setiembre. Sin embargo, MODIS registra el valor máximo el 19 de agosto, esta diferencia podría ser debido a que el AOD MODIS se mide con una longitud de onda de $550 \mathrm{~nm}$, mientras el AOD AERONET se mide a $440 \mathrm{~nm}$, el otro factor que puede generar esta diferencia es que AERONET mide un punto, mientras que MODIS lo hace en un rango de $25 \mathrm{~km}$, teniendo como centro la estación AERONET.

La Tabla 6 muestra la estadística mensual de la variación del AOD AERONET, done se observa que el mayor valor medio del AOD (0.235) se registran en el mes de agosto y el menor valor medio del AOD (0.094) se registró en el mes de julio. El máximo absoluto del AOD (0.825) se registró el 19 de setiembre y mínimo absoluto del AOD se registró 28 de julio.

\begin{tabular}{lcccl}
\hline $\mathrm{N}=10029$ & Media & $\sigma$ & Mínimo & Máximo \\
\hline AOD & 0.159 & 0.073 & 0.031 & 0.825 \\
EA & 1.624 & 0.198 & 0.239 & 2.144 \\
\hline
\end{tabular}

Tabla 5: Estadística general para AOD (440 nm) y EA (440$870 \mathrm{~nm}$ ) para el periodo julio-octubre. Donde $\mathrm{N}$ es el número de mediciones 


\begin{tabular}{lcccr}
\hline Mes & Medias & $\sigma$ & Mínimo & Máximo \\
\hline Jul & 0.094 & 0.041 & 0.031 & 0.298 \\
Ago & 0.235 & 0.112 & 0.059 & 0.666 \\
Set & 0.191 & 0.094 & 0.050 & 0.825 \\
Oct & 0.118 & 0.043 & 0.047 & 0.509 \\
\hline
\end{tabular}

Tabla 6: Estadística mensual para el AOD (440 nm) para el periodo julio-octubre.

La Figura 6 muestra la variación temporal del EA, el mínimo valor del AE (0.239) se registró el 3 de octubre y maximo absulto del AE (2.144) se registro el 10 octubre.

\begin{tabular}{lcccr}
\hline Mes & Medias & $\sigma$ & Mínimo & Máximo \\
\hline Jul & 1.519 & 0.170 & 0.572 & 1.962 \\
Ago & 1.737 & 0.165 & 0.637 & 2.050 \\
Set & 1.659 & 0.182 & 0.360 & 2.019 \\
Oct & 1.582 & 0.276 & 0.239 & 2.144 \\
\hline
\end{tabular}

Tabla 7: Estadística general para el EA (440-870 nm) para el periodo julio-octubre.

La Tabla 7 muestra la estadística mensual de la variación del EA AERONET, se observa que el mayor valor medio del EA (1.737) se registran en el mes de agosto y el menor valor medio del EA (1.519) se registró en el mes de julio. El máximo absoluto del EA (2.144) se registró octubre.

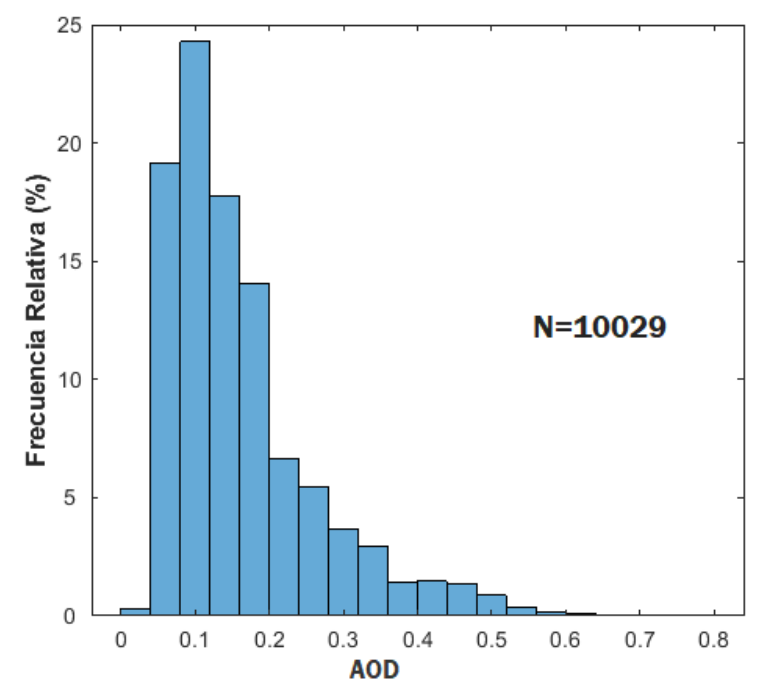

Figura 7: Distribución de frecuencia del AOD AERONET para $440 \mathrm{~nm}$ de longitud de onda.

La Figura 7 muestra que la mayor distribución de frecuencia del AOD es $24.3 \%$, con un valor de AOD de 0.100. El $75 \%$ del AOD varía entre 0.060 y 0.180 , en este rango se encuentran la mayor cantidad de valores del AOD. El valor más alto del AOD (0.620) representa tan sólo el $0.1 \%$ del total de la distribución de frecuencias del AOD.

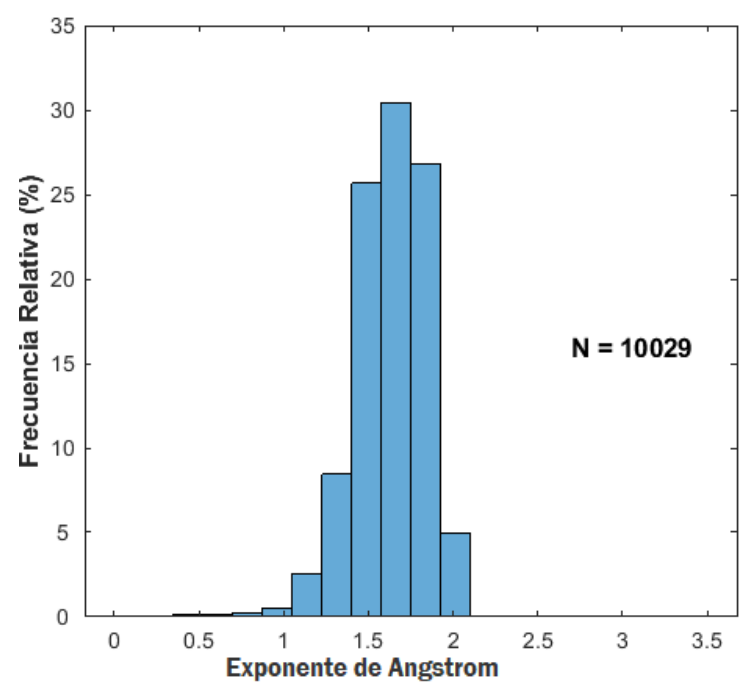

Figura 8: Distribución de frecuencia del EA AERONET para una longitud de onda que varía entre 449-870 $\mathrm{nm}$.

La Figura 8 muestra que la mayor distribución de frecuencia del EA es $30.5 \%$, con un valor de EA de 0.1663 . El $83 \%$ del EA varía entre 1.487 y 1.837 . El valor más alto del EA (2.012) representa tan sólo el $4.9 \%$ del total de la distribución de frecuencias del EA.

\subsection{Clasificación de tipos de aerosoles}

El método utilizado para la clasificación de aerosoles es el diagrama de dispersión del AOD versus EA (Verma et al., 2015) [Ver15], donde el AOD proporciona información sobre carga de aerosoles y EA sobre sus tamaños; el análisis conjunto de estas dos variables permite una mejor interpretación de datos (Estevan et al., 2019) [Est19].

Para la clasificación de aerosoles, se establecen rangos para definir los tipos de aerosoles. Las selecciones de un umbral de rangos se establecen de acuerdo a las condiciones específicas de cada sitio de medición y su entorno (Estevan et al., 2019) [Est19]. Para establecer umbrales iniciales y la clasificación de los aerosoles se utilizó la metodología de Estevan et al., (2019) [Est19].

Para la clasificación de aerosoles se han considerado seis tipos: Continental (CNT), Polvo (PVO), Marítimo (MAR), Biomasa (BIO), Urbano (URB) y Mezcla (MZC).

En la Tabla 8 y Figura 9a se muestra la estadística mensual de los tipos de aerosoles sobre el observatorio de Huancayo. El tipo BIO alcanza su mayor porcentaje en el mes de agosto $(81.36 \%)$ seguido por el mes de setiembre $(65.21 \%)$, esto debido a que la mayor cantidad de incendios forestales generados en dichos meses. Tal como se muestra en la Tabla 4, la mayor parte de los aerosoles 
de tipo BIO que llegan al observatorio de Huancayo proceden de la Amazonía Peruana y en menor porcentaje de Brasil y Bolivia, tal como se muestra en la Figura 10. El otro tipo de aerosol con alto porcentaje mensual es el tipo CNT, el cual alcanza su mayor porcentaje en el mes de agosto (83.93\%) seguido por el mes octubre $(74.80 \%)$

En la Tabla 8 y Figura 9b se muestra la estadística de los tipos de aerosoles en el perido julio a octubre. Los aerosoles de tipo CNT representan el $51.9 \%$ del total de casos.

\begin{tabular}{lllllll}
\hline Mes & BIO & MAR & PVO & MZC & URB & CNT \\
\hline Jul & 15.27 & 0.12 & 0.00 & 0.03 & 0.65 & 83.92 \\
Ago & 81.36 & 0.00 & 0.09 & 0.29 & 0.00 & 18.23 \\
Set & 65.21 & 0.31 & 0.18 & 0.06 & 0.31 & 33.87 \\
Oct & 21.55 & 0.74 & 1.18 & 0.31 & 1.42 & 74.80 \\
\hline
\end{tabular}

Tabla 8: Estadística porcentual mensual por tipos de aerosoles en el periodo julio a octubre del 2019 .

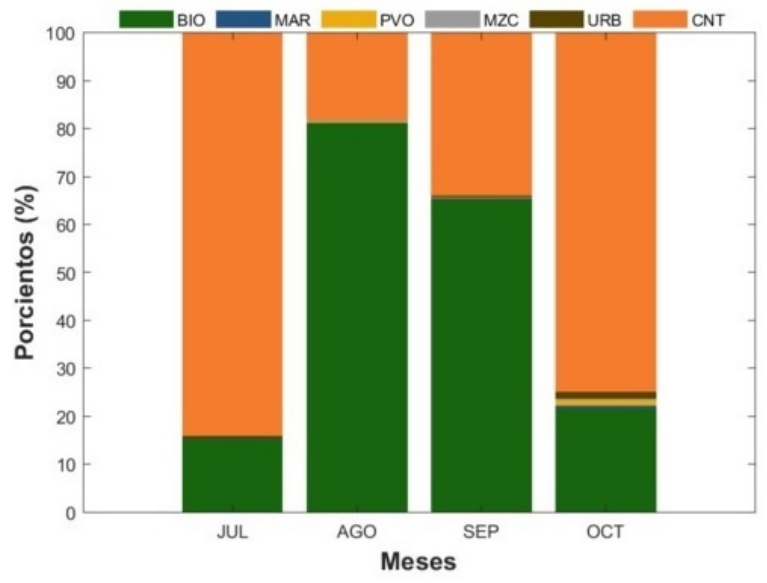

(a) Porcentaje de tipos de aerosoles por meses

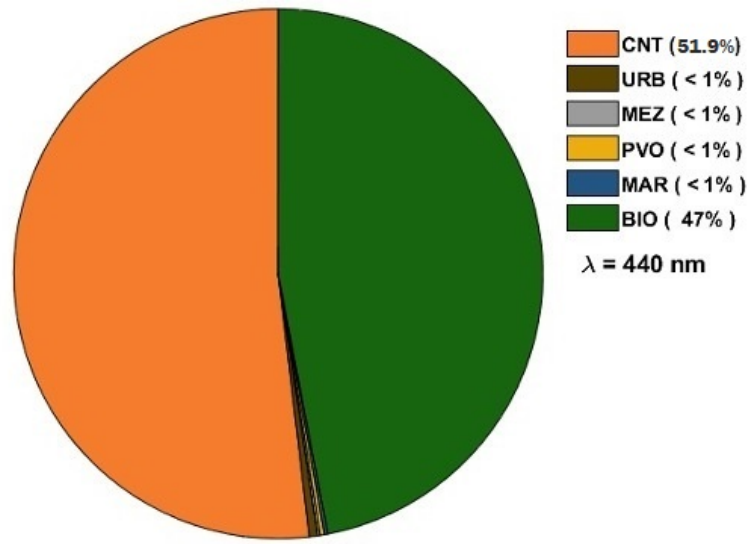

(b) Porcentaje de tipos de arosoles en el periodo

Figura 9: Porcentajes mensuales de la presencia de aerosoles por tipos en el Observatorio de Huancayo para el periodo de estudio.

\begin{tabular}{lcccccc}
\hline Tipo & AOD & $\sigma$ & EA & $\sigma$ & Frecuencia $(\%)$ & N (Número de casos) \\
\hline CNT & 0.091 & 0.025 & 1.531 & 0.170 & 51.9 & 5203 \\
URB & 0.067 & 0.020 & 1.830 & 0.100 & 0.5 & 50 \\
MZC & 0.193 & 0.064 & 0.924 & 0.065 & 0.2 & 17 \\
PVO & 0.230 & 0.117 & 0.503 & 0.144 & 0.2 & 25 \\
MAR & 0.107 & 0.016 & 0.637 & 0.131 & 0.2 & 21 \\
BIO & 0.240 & 0.098 & 1.742 & 0.165 & 47.0 & 4711 \\
\hline
\end{tabular}

Tabla 9: Estadística por tipos de aerosores, incluye el valor medio del AOD y EA, $\sigma$, el número de casos y la frecuencia de ocurrencia.

Los aerosoles CNT consisten principalmente en pequeñas partículas con altos valores EA (1.531), pero en el caso de AOD (0.091), es muy variable debido a su dependencia de si el aerosol está más o menos contaminado (Estevan et al., 2019) [Est19].

El segundo tipo de aerosol más registrado en Huancayo es el tipo BIO con $47.0 \%$ de todos los aerosoles medidos. Los aerosoles de tipo BIO al igual que el tipo $\mathrm{CNT}$, tiene valores AE altos, lo que significa que se constituye de partículas finas. El tipo BIO es el que modula el comportamiento de AOD en Huancayo, ya que el tipo BIO describe las mismas características mensuales del AOD, siendo máximo en el mes de agosto seguido por setiembre. Los aerosoles tipo BIO son consecuencia de la quema de biomasa de los incendios generados principalmente en Perú, pero también en menor medida como resultado de los incendios forestales registrados en Brasil y Bolivia, tal como se observa en la Figura 9.

Los aerosoles de tipo URB representan el $0.5 \%$ de todos los tipos de aerosoles. El tipo URB tiene valor medio 
EA alto (1.830), ligados a partículas muy pequeñas, son consecuencias de actividades antropogénicas e industriales. Con respecto al valor medio AOD (0.067), se observa que es menor que de los otros tipos de aerosoles.

Los aerosoles de tipo MAR representan el $0.2 \%$ de todos los tipos de aerosoles medido en Huancayo. Estos aerosoles poseen bajos valores AOD (0.107) y EA (0.637), que están relacionados con partículas gruesas.

Los aerosoles de tipo PVO representan el $0.2 \%$ de todos los aerosoles medidos en Huancayo. Éste tipo de aerosoles tiene valores EA (0.530) bajos al igual que el tipo MAR, por tanto, está formado por partículas gran- des. Sin embargo, el tipo PVO tiene valores elevados de AOD (0.230) al igual que tipo BIO. Las fuentes de aerosoles de tipo PVO son los terrenos agrícolas cercanas al observatorio y el desierto costero central del Perú (Estevan et al., 2019) [Est19].

Los aerosoles de tipo MZC, que representa el $0.2 \%$ de todos los tipos de aerosoles medido en Huancayo. Dado que es muy difícil determinar el límite exacto entre los aerosoles de tipo BIO y PVO, el tipo MZC se clasifica en la zona de transición de estos tipos de aerosoles (Estevan et al., 2019) [Est19].
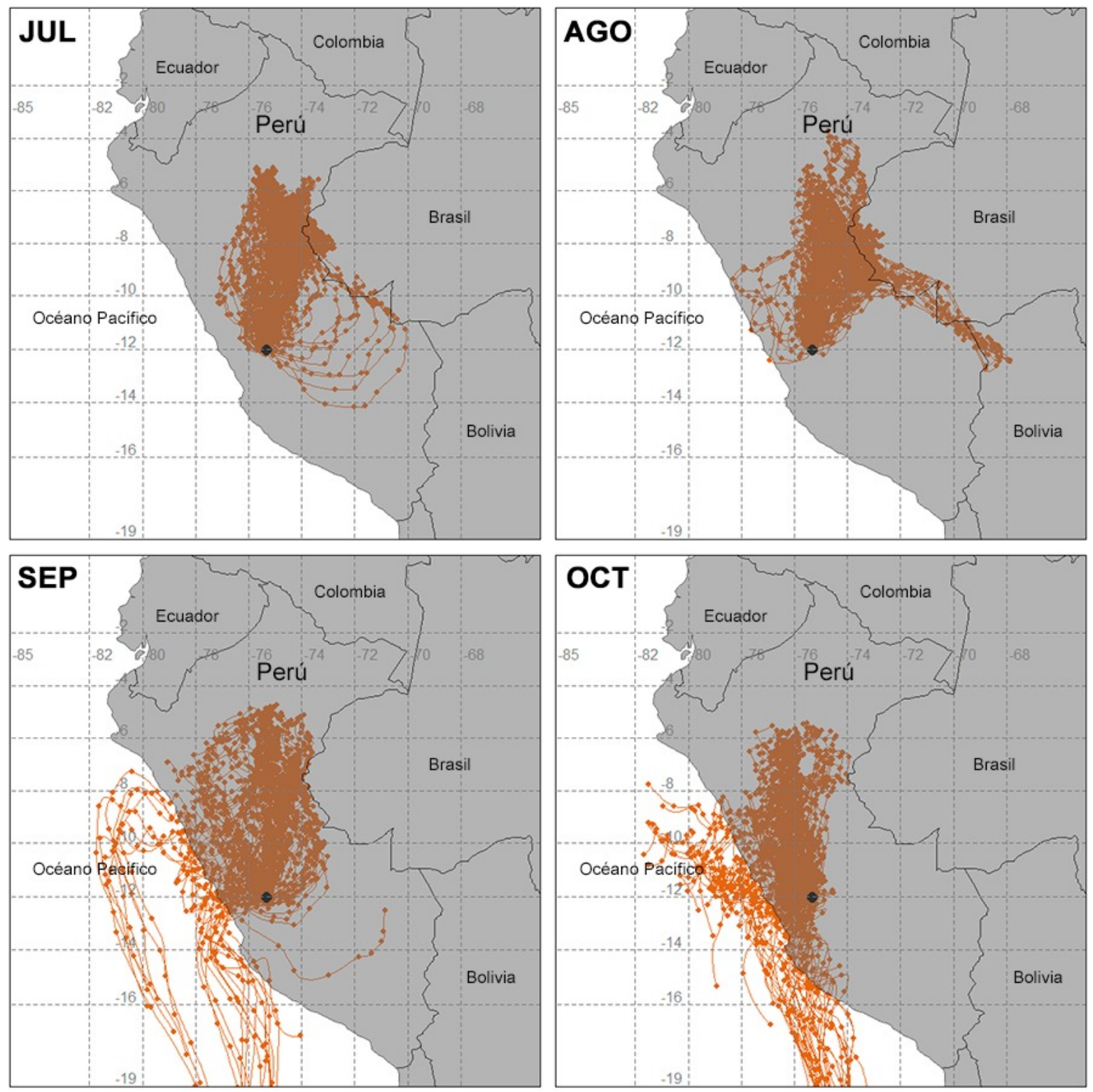

Figura 10: Trayectoria mensual de masas de aire. El punto negro representa representa al Observatorio de Huancayo. 
Los resultados aquí obtenidos sobre los porcentajes de tipos de aerosoles registrados en el Observatorio de Huancayo difieren a los obtenidos por Moya et al. (2017) [Moy17]. Moya et al. (2017) [Moy17] realizaron un estudio en el periodo junio a agosto del 2015, en su resultado muestran que el porcentaje de tipo MAR sobre el observatorio de Huancayo para los meses de junio, julio y agosto respectivamente son: $13 \%, 20 \%$ y $6 \%$, y el porcentaje de aerosol de tipo CNT sobre el observatorio de Huancayo para los meses de junio, julio y agosto respectivamente son: $84 \%, 72 \% 44 \%$. La diferencia entre nuestro resultado y el obtenido por Moya et al. (2017) [Moy17] demuestra la gran variabilidad porcentual de los tipos de aerosoles.

\subsection{Análisis de regiones fuentes de aerosoles mediante HYSPLIT}

Para determinar el origen de los aerosoles que llegan al Observatorio de Huancayo, se realizó un estudio de retrotrayectoria considerando 120 horas hacia atrás y una altitud de $200 \mathrm{~m}$ sobre el Observatorio de Huancayo. Las retrotrayectorias de HYSPLIT mostradas en la Figura 10 revelan diveras trayectorias de masas de aire, en el mes de julio los aerosoles se originan principalmente en el Perú y en menor medida en Brasil, en el mes de agosto los aerosoles se orginan en Perú, Brasil y Bolivia. Sin embargo, en los meses de setiembre y octubre los aerosoles se originan en el territorio peruano y el océno Pacífico. En la Figura 11 se muestra las trayectorias de masas de aire, en términos de clusters de trayectorias, expresada en porcientos, se oberva que la mayor cantidad de masas de aire que llegan al Observatorio de Huancayo se originan principalmente en la amazonía peruana, estas masas de aire traen consigo los aerosoles tipos BIO orginados por incendios forestales. El $16.8 \%$ de las masas de aire que llegan al Observatorio de Huancayo, se originan en el Océano Pacífico, estas masas de aire atraviesan los desiertos costeros de la Costa central del Perú, por lo que transportan aerosoles de tipo MAR y PVO (originados por vientos sobre la costa).

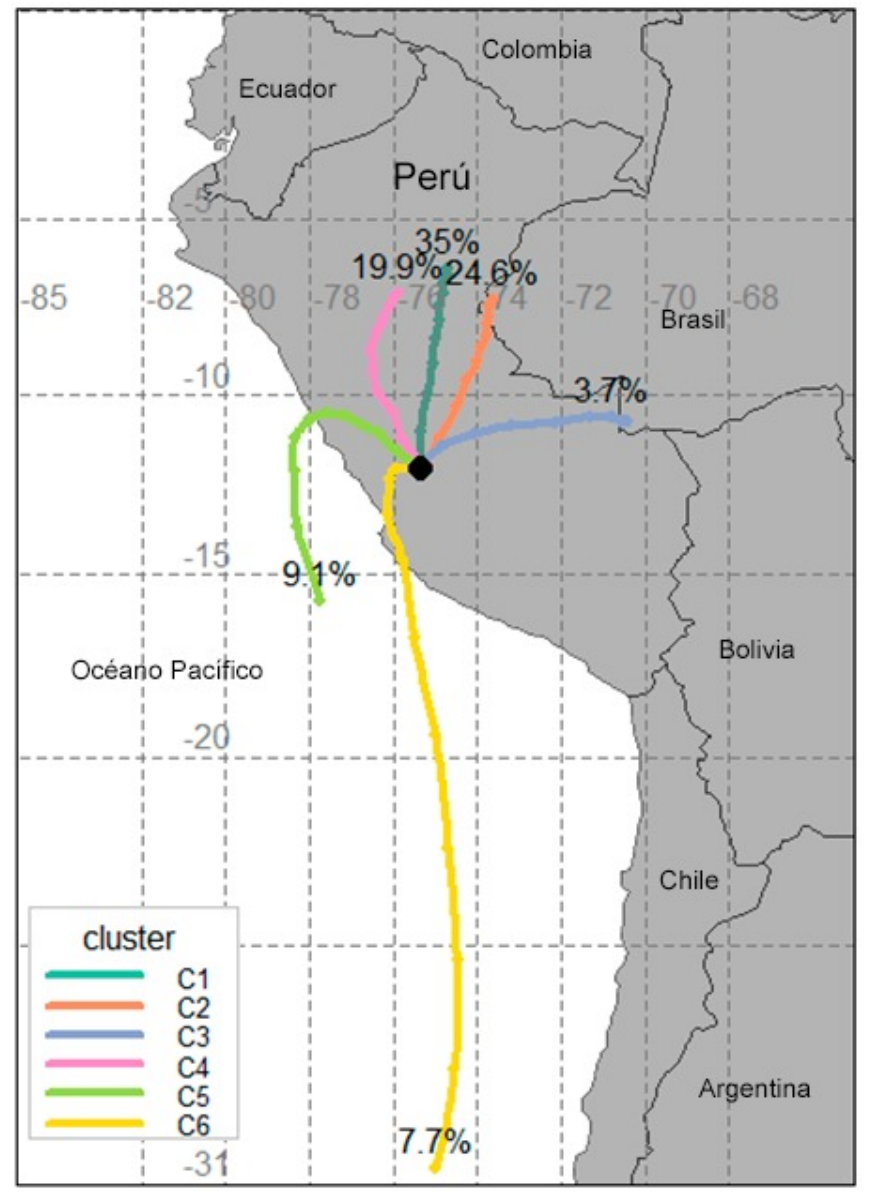

Figura 11: Trayectoria de clusters expresados en porcientos de masas de aire. 


\subsection{Intercomparación del AOD MODIS con AERONET en el periodo julio octubre del año 2019}

Para la intercomparación se ha utilizado la ecuación 1. En la Tabla 10 y Figura 12 se muestra la intercomparación del AOD MODIS con AERONET. Para la intercomparación Aqua con AERONET, el coeficiente de correlación (R) es 0.794 , BIAS es 0.025 ; lo cual indica que MODIS subestima el AOD, esta subestimación es debido a que las longitudes de onda de MODIS y AERONET no son indénticas. Para la intercompración Terra con AERONET, el coeficiente de correlación (R) es 0.796, BIAS es 0.019. Los altos valores $\mathrm{R}$ obtenidos en la intercomparación, indican que MODIS realiza un bue-

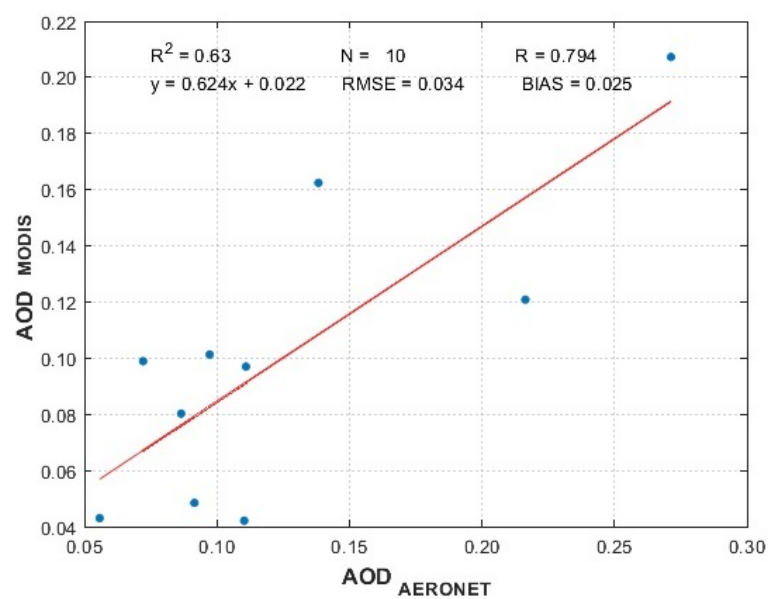

(a) Aqua

Figura 12: Frecuencia del EA AERONET.

\section{Conclusiones}

En este artículo hemos caracterizado los aerosoles en la estación AERONET de Huancayo. En nuestro análisis determinamos el AOD MODIS, AOD y EA AERONET, los tipos de aerosoles, los focos de incendios y las retrotrayectorias.

En el periodo de estudio se encontró que el valor medio, mínimo y máximo del AOD Terra es mayor que el OAD Aqua. Los valores máximos absolutos del AOD MODIS se registraron en el mes de agosto.

La mayor cantidad de incendios forestales se registraron en los meses de agosto (62 629) y setiembre (60 957); éste es el mes donde ocurrió también la máxima emisión de $P M_{10}\left(9.58 \times 10^{4} \mathrm{~kg} /\right.$ día $)$.

El máximo absoluto del AOD AERONET se registró en el mes de setiembre, como consecuencia de las mayores emisiones. Sin embargo, el mayor valor del EA se na recuperación del AOD en el periodo de estudio. Los valores $R$ obtenidos en la investigación son superiores a los obtenidos por Boiyo et al. (2019) [Boi17] y Floutsi et al. (2019) [Flo16]. Boiyo et al. (2019) [Boi17] obtuvo un valor $\mathrm{R}$ para las ciudades de Malindi, Mbita, Nairobi y Kibale, respectivamente, $0.64,0.67,0.34$ y 0.60 . Floutsi et al. (2019) [Flo16] obtuvo un valor $\mathrm{R}$ de 0.766 sobre el Mediterráneo.

\begin{tabular}{lcccc}
\hline Satélite & $\mathrm{R}$ & $\mathrm{BIAS}$ & $\mathrm{RMSE}$ & $\mathrm{N}$ \\
\hline Aqua & 0.794 & 0.025 & 0.034 & 10 \\
Terra & 0.796 & 0.019 & 0.063 & 10 \\
\hline
\end{tabular}

Tabla 10: Intercomparación del AOD MODIS con AERONET en el periodo julio octubre del año 2019.



(b) Terra

registró en el mes de octubre.

Los tipos de aerosoles predominantes en la Estación AERONET de Huancayo son CNT (51.9\%) y BIO (47.0\%). Nuestro resultado sobre el porcentaje de tipos de aerosoles es diferente a lo obtenido por Moya et al. (2017), la diferencia más notable está en los aerosoles tipo CNT y MAR.

La mayor cantidad de masas de aire que llegan al Observatorio de Huancayo se originan principalmente en la amazonía peruana, estas masas de aire traen consigo los aerosoles tipos $\mathrm{BIO}$ originados por incendios forestales.

Al intercomparar el AOD MODIS con el AOD AERONET, se obtuvieron valores del coeficiente de correlación $(\mathrm{R})$ altos, los cuales indican que MODIS realiza un buena recuperación del AOD. Por tanto, se puede utilizar los datos MODIS para caracterizar el AOD en las zonas que estén a una distancia de $25 \mathrm{~km}$ del Observatorio de Huancayo. 


\section{Agradecimientos}

La presente ivestigación se desarrolló en el marco del proyecto Magnet del Instituto Geofísico del Perú (IGP) Fortalecimiento de la linea de investigación en Física y
Microfísica de la Atmósfera, financiado por el Fondo Nacional de Desarrollo Científico, Tecnológico y de Innovación Tecnológica-FONDECYT del CONCYTEC (Convenio $N^{0} 010-2017$-FONDECYT).

\section{Referencias}

[Art09] Artaxo, P., Rizzo, L., Paixao, M., Lucca, S., Oliveira, P., Lara, L., Wiedemann, K., Andreae, M., Holben, B., Schafer, J., Correia, A., Pauliquevis, T. (2009). Aerosol Particles in Amazonia: Their Composition, Role in the Radiation Balance, Cloud Formation, and Nutrient Cycles. Amazonia and Global Change Geophysical Monograph Series 186 Copyright 2009 by the American Geophysical Union, 10.1029/2008GM0007r8

[Bil13] Bilal, M., Nichol, J., Bleiweiss, M., Dubois, D. y Rse., J. (2013). A simplified high resolution MODIS aerosol retrieval algorithm (SARA) for use over mixed surfaces. RRemote Sens. Environ., 136(), pp 135-145.

[Bha17] Bhat, M., Romshoo, S y Beig, G. (2017). Aerosol black carbon at an urban site-Srinagar, Northwestern Himalaya, India: Seasonality, sources, meteorology and radiative forcing. Atmospherical Environment, $165(1)$, pp 336-348.

[Bil14] Bilal, M., Nichol, J. y Chan, P. (2014). Validation and accuracy assessment of a simplified aerosol retrieval algorithm (SARA) over Beijing under low and high aerosol loadings and dust storms. Remote Sens. Environ., 153(), pp 50-60.

[Bri19] Bright, J. y Guyemard, C. (2019). Climatespecific and global validation of MODIS Aqua and Terra aerosol optical depth at 452 AERONET stations. Solar Energy, 183(), pp 549-605.

[Boi17] Boiyo, R., Raghavendra, K., Zhao, T. y Bao, Y. (2017). Climatological analysis of aerosol optical properties over east Africa observed from spaceborne sensors during 2001-2015. Atmospherical Environment, 152(), pp 298-313.

[Dua19] Duan, S., Li, Z., Li, H., Gottsche, F., Wu, H., Zhao, W., Leng, P., Zhang, X., Coll, C. (2019). Validation of Collection 6 MODIS land surface temperature product using in situ measurements. Remote Sensing of Environment, 225(), pp 16-29.

[Est14] Esteve, A., Estellés, V., Utrillas, M. y MartínezLozano, J. (2014). Analysis of the radiative forcing over a Mediterranean urban coastel site. Atmospheric Research., 137(1), pp 195-204.

[Est19] Estevan, R., Martínez-Castro, D., Suarez-Salas, L., Moya, A., Silva, Y. (2019). First two and a half years of aerosol measurements with an AERONET sunphotometer at the Huancayo Observatory, Peru. Atmospheric Environment, 3(), 10037

[Fer17] Fernández, A., Molero, F., Salvador, P., Revuelta, A., Becerril-Valle, M., Gomez-Moreno, F., Artíñano, B., Pujadas, M. (2017). Aerosol optical, microphysical and radiative forcing properties during variable intensity dust events in the Iberian Peninsula. Atmospheric Research, 196(), pp 129-141.

[Flo16] Floutsi, A., Korras-Carraca, M., Matsoukas, C., Hatzianastassiou, N., Biskos y G. (2016). Climatology and trends of aerosol optical depth over the Mediterranean basin during the last 12 years (20022014) base don collection 006 MODIS-Aqua. Science of the Total Environment, 5511(), pp 292-303.

[Hsu13] Hsu, N., Jeong, M., Bettenhausen, C., Sayer, A., Hansell, R., Seftor, C, Huang, J, Tsay, S. (2013)). Enhanced Deep Blue aerosol retrieval algorithm: The second generations. J. Geophysics. Res. Atmos., 118(9), pp 9 296-9 315.

[IPC13] Intergovernmental Panel on Climate Change (IPCC), 2013. Climate change 2007: The Physical Science Basic: Contribution a Working Group I to the Fourth Asessment Report of the Intergovernmental Panel Climate Change. Chapter 2..

[Lev13] Levy, R., Mattoo, S., Muchak, L., Reemr, 1., Sayer , A., Patadia, F. y Hsu, N. (2013). The darkland MODIS collection 5 aerosol retrieval: algorithm development and product evaluation. Atmos. Meas. Teach., 6(), pp 2989-3034.

[Lev13] Levy, R., Mattoo, S., Muchak, L., Reemr, l., Sayer , A., Patadia, F. y Hsu, N. (2013). The collection 6 MODIS Aerosol products over Land and Ocean. Atmos. Meas. Teach., 6(), pp 2989-3034.

[Kop18] Koplitz, S., Nolte, C., Pouliot, G., Vukovich, J., Beidler, J. (2018). Influence of uncertainties in burned area estimates on modeled wildland fire PM2.5 and ozone pollution in the contiguous U.S. Atmospheric Environment, 191 (), pp 328-339. 
[Man20] Mangla, R., Indu, J. y Chakra, S. (2020). Intercomparison of multi-satellites and Aeronet AOD over Indian Region. Atmospheric Research, 240(), 104950 .

[Mao19] Mao, Q., Zhang, H., Chen, Q., Huang, C., Yuan, Y. (2019). Satellite-based assessment of direct aerosol radiative forcing using a look-up table established through AERONET observations. Infrared Physics \& Technology, 102(), 103017.

[Mao18] Mao, Q., Huang, C., Zhang, H., Chen, Q., Yuan, Y. (2018). Aerosol optical properties and radiative effect different weather conditions in Harbin, China. Infrared Physics \& Technology, 89(), pp 304-314.

[Moy17] Moya, A., Estevan, R. y Yuli R. (2017). Determinación de la presencia de partículas $\left(P M_{10}\right)$ en Perú producidas por quema de biomasa con ayuda de modelos numéricos. Rev. Int. Contam. Ambie., 33(1), pp 99-108.

[Nai14] Nair, V., Babu, S., Moorthy, K. y Satheesh, S. (2014). Implications of multiple ascattering on the assessment of black carbon aerosol raditive forcing. Journal of quantitative spectroscopy $\& 5$ radiative transfer., 148(), pp 134-140.

[Oso12] Osores, F., Rojas, J., Manrique, C. (2012). Minería informal e ilegal y contaminación con mercurio en Madre de Dios: Un problema de salud pública. . Acta Med per Educativa Universitaria, 29(), pp 3842.

[Say14] Sayer, A., Munchak, L., Hsu, N., Levy, R., Bettenhausen, C., Jeong, M. (2014). MODIS Collecction 6 aerosol products: Comparison between Auqa's e-deep Blue, Dark Target, and "merged" data sets, and usage recommendations. Journal of Geophysical Research: Atmospheres, 119(13), pp 13 965-13989.

[Sca18] Scanes, C. y Toukhsati, S. (2018). Animals and Human Society. Academic Press(), pp 541-482.

[Sha19] Shaik, Yogesh, K., Mitra1, D., Singh, A., Chandola, H., Sateesh, M., Babu, S., Chauhan, P. (2019). Impact of biomass burning on regional aerosol optical properties: A case study over northern India. Journal of Environmental Management, 244(1), pp 328-343.

[Sri18] Srivastava, A., Bisht, D., Singh, S., Kishore, N., Soni, V., Singh, S., Tiwari, S. (2018).Scattering and absorption characteristics of aerosols at an urban megacity over IGB: Implications to radiative forcing. Atmospherical Research, $205($ ), pp 107-117.
[Ste20] Stefan, S., Voinea, S. y Iorga., G. (2020). Study of the aerosol optical characteristics over the Romanian Black Sea Coast using AERONET data. Atmospheric Pollution Research, 11(7), pp 1165-1178.

[Sua17] Suarez, L., Torres, C., Helmig, D. y Hueber, J. (2017). Medición y análisis del aerosol de carbono negro en el observatorio de Huancayo, Perú. Revista boliviana de física, 30(), pp 7-15.

[Sun17] Sun, K., Chen, X., Wang, J., Zhang, T. y Zhu, Z. (2017). Investigations of air quality over the largest city in the central China using high resolution satellite derived aerosol optical deph data. Atmospheric pollution research, $30(40)$, pp 1-10.

[Ver15] Verma, S. (2015). A new classification of aerosol source and tipes as measured over Jaipur, India. Aersosol and tipes Air Quality Research, 15(), pp $985-993$

[Viv14] Vivanco, S. (2014). Variabilidad temporal de aerosoles atmosféricos en Huancayo. Apunt. cienc. soc., 04(01), pp 57-68.

[Von20] Vongruang, P. y Pimonsree, S. (2020). Biomass burning sources and their contributions to PM10 concentrations over countries in mainland Southeast Asia during a smog episode. Atmospheric Environment, 228(), 117414.

[Wan19] Wang, M.He, G., Zhang, Z., Wang, G., Wang, Z., Yin, R., Cui, S., Wu, Z., Cao, X. (2019).A radiance-based split-window algorithm for land surface temperature retrieval: Theory and application to MODIS data. Int $J$ App Earth Obs Geoinformation, 76(1), pp 204-217.

[Wan14] Wan, Z. (2014). New refinements and validation of the collection-6 MODIS land-surface temperature/emissivity product. Remote Sensing of Environment, $140(1)$, pp 36-45.

[Wei19] Wei, J., Li, Z., Peng, Y. y Sun, L. (2019). MODIS Collection 6.1 aerosol optical depth products over land and ocean: validation and comparison. Atmospheric Environment, 201(), pp 428-440.

[Wie11] Wiedinmyer, C., Akagi, S., Yokelson, R., Emmons, L., Al-Saadi, J., Orlando, J., Soja, A. (2011)). The Fire Inventory from NCAR (FINN): a high resolution global model to estimate the emissions from open burning. Geosci. Model Dev., 4(), pp 625-641.

[Xu20] Xu, X., Xie, L., Yang, X., Wu, H., Cai, L., Qi, P. (2020). Aerosol optical properties at seven AERONET sites over Middle East and Eastern Mediterranean Sea. Atmospheric Environment, 243(), pp 117884 . 
[Yu17] Yu, X., L ü, R., Liu, C., Yuan, L., Shao, Y., Zhu, B. y Lei, L. (2017). Seasonal variation of columnar aerosol optical properties and radiative forcing over
Beijing, China. Atmospherical Environment, 166(), pp 340-350. 